\title{
Hadron production within a full transport approach with statistical hadronization mechanism at RHIC and LHC en- ergies
}

\author{
Giuseppe Galesi ${ }^{1,2}$, Salvatore Plumari ${ }^{1,2}$, and Vincenzo Greco ${ }^{1,2}$ \\ ${ }^{1}$ Department of Physics and Astronomy, University of Catania, Via S. Sofia 64, I-95123 Catania, Italy \\ ${ }^{2}$ Laboratori Nazionali del Sud, INFN-LNS, Via S. Sofia 62, I-95123 Catania, Italy
}

\begin{abstract}
We present for the first time results on final hadron production, with and without strangeness content, in Ultrarelativistic Heavy Ion Collisions at RHIC and LHC center of mass energies obtained combining a full 3+1D relativistic Boltzmann transport approach with a statistical hadronization mechanism. The non-perturbative interaction between quarks and gluons is described by means of a quasi-particle approach that permits to have an Equation of State close to lattice QCD. The resulting framework naturally includes both shear and bulk viscous effects. The $3+1 \mathrm{D}$ full transport evolution is converted to hadrons by mean of a realistic freeze-out hypersurface considering all known hadron resonances and by performing resonance decays. We present results on charged-hadron multiplicity, identified-particle spectra and identified-particle elliptic flow of $\pi, K$ and $p$ produced at RHIC and LHC energies for different centralities.
\end{abstract}

\section{Introduction}

Relativistic viscous hydrodynamics is able to describe very well many observables in Ultra Relativistic Heavy Ion Collisions (uRHICs) such as final hadron spectra and elliptic flow in the low $p_{T}$ region [1-6]. Usually, such an approach makes use of a statistical hadronization mechanism [7-9] which converts the information contained in the energy-momentum tensor to final hadrons applying the CF (Cooper-Frye) formula [10] on a freeze-out hypersurface. This framework allows to put constraints on QGP properties: they are fixed from the beginning of a simulation and drive the dynamical evolution. Anyway, in order to transfer the viscous correction information $\delta T^{\mu v}$ to the final hadron particle distribution function an ansatz is needed for the deviation $\delta f$. Further, it does not allow for arbitrary far from-equilibrium initial conditions. A transport approach can self-consistently overcome both these problem because the one-particle distribution function is always known during the dynamical evolution. For these reasons we developed a transport approach at fixed $\eta / s$ which permits to have direct comparison with viscous hydrodynamics and to investigate specific dissipation properties of QGP. In order to test the validity of our approach we perform statistical hadronization on a hydro-like freeze-out hypersurface at fixed temperature extracted with our code and we directly compare the resulting hadron observables with experimental data. 


\section{The transport equation and the statistical hadronization}

In our approach, we describe the quark-gluon plasma (QGP) by solving Relativistic Transport Boltzmann (RTB) equations developed to study the dynamics of the matter created in uRHICs [11-15]:

$$
\left(p^{\mu} \partial_{\mu}+\frac{1}{2} \partial_{i} m^{2} \partial_{(p)}^{i}\right) f=C[f],
$$

$C[f]$ is the collision integral for $2 \rightarrow 2$ collision processes. In our approach non-pertubative effects are encoded by using a quasi-particle model (QPM) with thermal masses determined in order to reproduce lattice QCD equation of state [16]. The collision integral is gauged to the desired $\eta / s$ through the Chapmann-Enskog approximation and is computed by means of a stochastic algorithm $[17,18]$. The energy-momentum tensor is evaluated during the dynamical evolution of $f$ on a $\left(x, y, Y_{s}\right)$-grid, with $Y_{s}$ the longitudinal space rapidity, according to the formula

$$
T_{c}^{\mu \nu}=\frac{1}{N_{\mathrm{test}} V_{c}} \sum_{i \in c} \frac{p_{i}^{\mu} p_{i}^{v}}{p_{i}^{0}},
$$

where $N_{\text {test }}$ is the number of test particles per real particle, $V_{c}$ the cell volume, $p_{i}^{\mu}$ the test particle 4-momentum and being the sum over all test particles in the $c$-cell. From that we self-consistently extract the local temperature $T$, the thermal mass and local flow $u^{\mu}$. We then interpolate a freeze-out hypersurface $\Sigma$ at fixed temperature and we give it in input to THERMINATOR 2 code [19] which performs statistical hadronization task. It is a MonteCarlo event generator that produces final hadrons by using the following CF formula:

$$
p_{h}^{0} \frac{\mathrm{d}^{3} N_{h}}{\mathrm{~d} p^{3}}=\left(2 s_{h}+1\right) \int_{\Sigma} \mathrm{d} \Sigma_{\mu} p_{h}^{\mu} f_{h},
$$

being $\frac{\mathrm{d}^{3} N_{h}}{\mathrm{~d} p^{3}}$ the spectrum for the $h$-resonance, $s_{h}$ the relative spin quantum number and $f_{h}$ the following equilibrium distribution:

$$
f_{h}=\frac{1}{(2 \pi)^{3}}\left(\exp \left(\frac{p_{h, \mu} u^{\mu}}{T}\right) \pm 1\right)^{-1}
$$

where the sign is chosen according to the statistics of the resonance. Customizations of this formula which use modified versions of the distribution function to take into account local deviation from equilibrium are of course possible but for a first test of our approach we consider Fermi-Dirac and Bose-Einstein distributions only. All known hadronic resonances from the SHARE package [20] are considered for primordial particle production on $\Sigma$ and resonance decays are subsequently performed on unstable particles. In this way it is potentially possible to extract all final hadron observables to be compared with the available data from RHIC and LHC experimental collaborations in order to fix the free parameters, which are the initial central temperature $T_{0}$ for the most central collision, the freeze-out temperature $T_{\mathrm{FO}}$ and $\eta / s$. In particular we studied AuAu collisions at RHIC with a center of mass energy $\sqrt{s}=200 \mathrm{AGeV}$ and $\mathrm{PbPb}$ collision at LHC with $\sqrt{s}=2.76 \mathrm{ATeV}$.

\section{Results}

We show only results for semi-peripheral LHC collisions. We initialize the system at fixed proper time $\tau_{0}=0.25 \mathrm{fm} / \mathrm{c}$. Initialization of test particles space coordinates is performed in the transverse plane by scaling particle number density or energy density according to an 
OGM (Optical Glauber Model) with mixed contributions from number of wounded nucleons $N_{\text {part }}$ and binary collisions $N_{\text {coll }}$ and with a tilted function of rapidity. Instead, the longitudinal profile has a plateau in the mid rapidity region with gaussian tails whose parameters are set to reproduce final pseudorapidity hadron yields. In momentum space we distribute according to a 3D isotropic thermal distribution with Bjorken flow. In Figure 1 on the left we show the mid-rapidity spectra for pion, kaon and proton (filled curves) obtained in a semi-peripheral LHC collision at $b=7.7 \mathrm{fm}$ with $T_{0}=670 \mathrm{MeV}, T_{\mathrm{FO}}=145 \mathrm{MeV}$ and $\eta / s=1 / 4 \pi$, and the corresponding ALICE data $[21,22]$ for the centrality class $20-30 \%$. The pion and kaon spectra are quite well reproduced over all the $p_{\mathrm{T}}$ region shown while protons are slightly underestimated above $1.5 \mathrm{GeV}$. We attribute this fact to the missing hadronic afterburner which can have a significant effect for heavier particles, and which could be taken into account in future works by using other more sophisticated numerical code like SMASH [23]. On
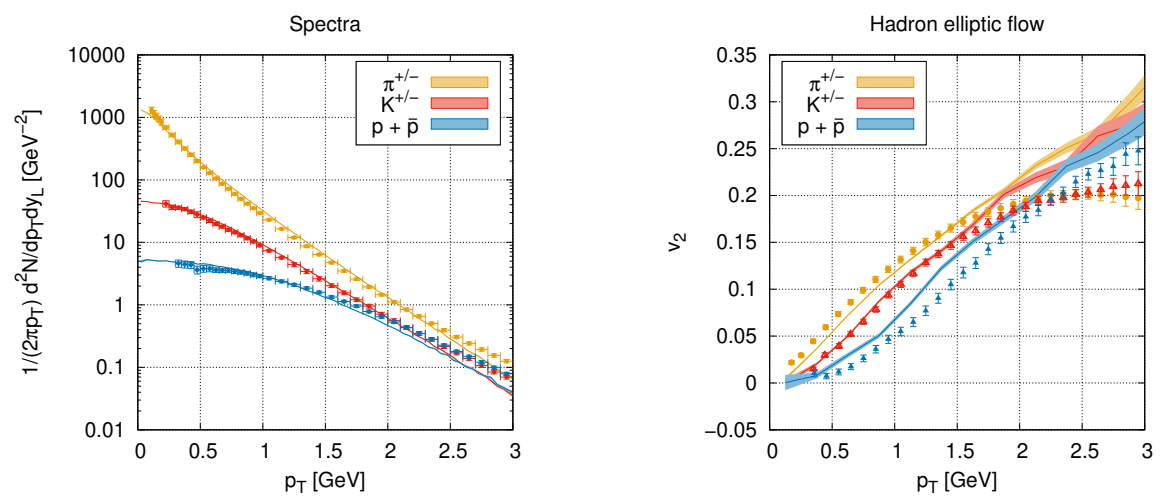

Figure 1. Left panel: Identified hadron spectra of $\pi$ (orange curve), $K$ (red line) and $p+$ anti- $p$ (blu curve) for $\mathrm{PbPb}$ collisons at $\sqrt{s}=2.76 \mathrm{TeV}$. Circle, triangles and square point are experimental data from ALICE collaboration taken from [21]. Right panel: elliptic flow $v_{2}$ for $\mathrm{Pb}+\mathrm{Pb}$ collision at $\sqrt{s}=$ $2.76 \mathrm{TeV}$ of $\pi, K, p$ for 20-30\% centrality cut and at mid-rapidity. Experimental data taken from [22]

the right of Figure 1 we show, for the same system, the differential elliptic flow caused by the initial space anisotropy. This final momentum anisotropy is due only to the shape of the freeze-out hypersurface and local flow information interpolated on it because we don't consider any non-equilibrium effect in the hadron distribution function. Nevertheless, we have a good agreement with experimental data. We have also observed a strong dependence of this quantity from the initial mixing factor of the OGM, which seems to suggest that a greater contribution should be considered from $N_{\text {coll }}$ at LHC energies.

\section{Conclusions}

In conclusion, we have shown that this framework is able to reproduce quite well final hadron observables and we believe that it is still possible a further fine tuning on the free parameters to have a better agreement with experimental data [24]. This validation test opens the way to many possible applications of this code. One of the most intriguing perspective is the possibility to make a direct comparison between a microscopic hadronization model like coalescence plus fragmentation $[25,26]$ with a macroscopic one like the SHM presented here using an underlying common framework. Furthermore, we are planning to investigate in the next future the isotropization and hydrodynamization processes of a strongly interacting 
system which starts with non-equilibrium initial conditions by looking for hydrodynamic attractors of a realistic massive gas.

\section{References}

[1] P. Huovinen, P.F. Kolb, U. Heinz, P.V. Ruuskanen, S. Voloshin, Phys. Lett. B503 58-64 (2001)

[2] T. Hirano, K. Tsuda, Nucl. Phys. A715 821-824 (2002)

[3] P.F. Kolb, U. Heinz (2003)

[4] B. Schenke, S. Jeon, C. Gale, Phys. Rev. C82 014903 (2010)

[5] U. Heinz, R. Snellings, Ann. Rev. Nucl. Part. Sci. 63, 123 (2013)

[6] M. Alqahtani, M. Nopoush, M. Strickland, Prog. Part. Nucl. Phys. 101, 204 (2018)

[7] J. Cleymans, H. Satz, Z. Phys. C 57, 135 (1993)

[8] F. Becattini, M. Gazdzicki, J. Sollfrank, Eur. Phys. J. C 5, 143 (1998)

[9] W. Florkowski, W. Broniowski, M. Michalec, Acta Phys. Polon. B33: 761-769 (2001)

[10] F. Cooper, G. Frye, Phys. Rev. D 10, 186 (1974)

[11] F. Scardina, M. Colonna, S. Plumari, V. Greco, Phys. Lett. B724 (2013) 296-300 (2012)

[12] M. Ruggieri, A. Puglisi, L. Oliva, S. Plumari, F. Scardina, V. Greco, Phys. Rev. C 92, 064904 (2015)

[13] A. Gabbana, S. Plumari, G. Galesi, V. Greco, D. Simeoni, S. Succi, R. Tripiccione, Phys. Rev. C 101, 064904 (2020) (2019)

[14] M.L. Sambataro, S. Plumari, V. Greco (2020)

[15] Y. Sun, S. Plumari, V. Greco, Eur. Phys. J. C 80, 16 (2020)

[16] S. Plumari, W.M. Alberico, V. Greco, C. Ratti (2011)

[17] S. Plumari, A. Puglisi, F. Scardina, V. Greco, Phys. Rev. C 86, 054902 (2012)

[18] Z. Xu, C. Greiner, Phys. Rev. C 79, 014904 (2009)

[19] M. Chojnacki, A. Kisiel, W. Florkowski, W. Broniowski (2011)

[20] G. Torrieri, S. Steinke, W. Broniowski, W. Florkowski, J. Letessier, J. Rafelski, Comput. Phys. Commun. 167, 229 (2005)

[21] J.A. et al. (ALICE Collaboration), Phys. Rev. C 93 (2016)

[22] A. Collaboration, JHEP 06 (2015) 190 (2014)

[23] J. Weil et al., Phys. Rev. C 94, 054905 (2016)

[24] G. Galesi, S. Plumari, V. Greco (In preparation)

[25] V. Greco, C.M. Ko, Acta Phys. Hung. A24 (2005) 235-240 (2004)

[26] V. Minissale, F. Scardina, V. Greco, Phys. Rev. C 92, 054904 (2015) 